\title{
Intramolecular C-H Activation Reactions of Molybdenacyclobutanes
}

\author{
Peter M. Graham, Miriam S. A. Buschhaus, and Peter Legzdins* \\ Department of Chemistry, The University of British Columbia, Vancouver, British Columbia, \\ Canada V6T 1 Z1
}

Supporting Information 


\section{Experimental Procedures}

All reactions and subsequent manipulations involving organometallic reagents were performed under anaerobic and anhydrous conditions either at a vacuum-nitrogen dual manifold or in an inertatmosphere dry box. Pentane, benzene- $d_{6}$, diethyl ether, and tetrahydrofuran (THF) were all dried over sodium benzophenone ketyl and were freshly distilled prior to use. Cyclopentene, cyclohexene, cycloheptene, and cyclooctene were all purchased from Aldrich, and were dried over sodium benzophenone ketyl, distilled, and stored in resealable glass vessels. $\mathrm{Cp}{ }^{\star} \mathrm{Mo}(\mathrm{NO})\left(\mathrm{CH}_{2} \mathrm{CMe}_{3}\right)_{2}$ (1) was prepared according to the published procedure. ${ }^{1}$ All other chemicals were purchased from Aldrich and used as received.

All IR samples were prepared as Nujol mulls sandwiched between $\mathrm{NaCl}$ plates, and their spectra were recorded on a Thermo Nicolet Model 4700 FT-IR spectrometer. NMR spectra were recorded at room temperature on Bruker AV-400 or Bruker AV-500 instruments, and all chemical shifts and coupling constants are reported in ppm and in Hertz, respectively. ${ }^{1} \mathrm{H}$ NMR spectra were referenced to the residual protio isotopomer present in $\mathrm{C}_{6} \mathrm{D}_{6}(7.16 \mathrm{ppm}) .{ }^{13} \mathrm{C}$ NMR spectra were referenced to $\mathrm{C}_{6} \mathrm{D}_{6}$ (128 ppm). Where necessary, ${ }^{1} \mathrm{H}-{ }^{1} \mathrm{H}$ COSY,${ }^{1} \mathrm{H}-{ }^{13} \mathrm{C} \mathrm{HMQC}$, and ${ }^{13} \mathrm{C}$ APT experiments were carried out to correlate and assign ${ }^{1} \mathrm{H}$ and ${ }^{13} \mathrm{C}$ NMR signals. Low-resolution mass spectra (El, $70 \mathrm{eV}$ ) were recorded by the staff of the UBC mass spectrometry facility using a Kratos MS-50 spectrometer. Elemental analyses were performed by Mr. Minaz Lakha of the UBC microanalytical facility.

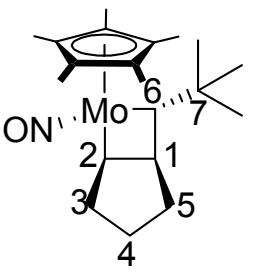

Complex 2A: A red solution of $\mathrm{Cp}{ }^{*} \mathrm{Mo}(\mathrm{NO})\left(\mathrm{CH}_{2} \mathrm{CMe}_{3}\right)_{2}(0.079 \mathrm{~g}, 0.196 \mathrm{mmol})$ in cyclopentene $(10 \mathrm{~mL})$ became orange after $26 \mathrm{~h}$. The final solution was then evaporated to dryness in vacuo and the residue was recrystalized from diethyl ether/pentane at $-30^{\circ} \mathrm{C}$ overnight to obtain $2 \mathrm{~A}$ as an orange powder $(0.039 \mathrm{~g}, 0.135$ $\mathrm{mmol}, 69 \%)$.

Anal. Calcd for $\mathrm{C}_{20} \mathrm{H}_{33}$ MoNO: C, 60.14; $\mathrm{H}, 3.51 ; \mathrm{N}, 8.33$. Found: $\mathrm{C}, 60.13 ; \mathrm{H}, 3.90 ; \mathrm{N}, 8.06$; IR (Nujol): $v_{\mathrm{NO}}=1559 \mathrm{~cm}^{-1} ;{ }^{1} \mathrm{H} \operatorname{NMR}\left(\mathrm{C}_{6} \mathrm{D}_{6}, 400 \mathrm{MHz}, 25^{\circ} \mathrm{C}, \delta\right): 7.94(1 \mathrm{H}, \mathrm{dd}(\mathrm{J}=18.7,8.3), \mathrm{C} 2 \mathrm{H}), 3.27$ (1H, d $(J=5.2), C 6 H), 2.42\left(1 \mathrm{H}\right.$, ddd $\left.(J=12.4,8.3,5.6), C 3 \mathrm{H}_{2}\right), 1.94\left(1 \mathrm{H}, \mathrm{m}\right.$ (overlapping), $\left.\mathrm{C} 4 \mathrm{H}_{2}\right), 1.88$ (1H, m (overlapping), C5 $\left.\mathrm{H}_{2}\right), 1.80\left(1 \mathrm{H}, \mathrm{m}\right.$ (overlapping), $\left.\mathrm{C}_{3} \mathrm{H}_{2}\right), 1.74\left(15 \mathrm{H}, \mathrm{s}, \mathrm{Cp} \mathrm{p}^{*}\right), 1.70(1 \mathrm{H}, \mathrm{m}$ (overlapping), $\left.\mathrm{C} 4 \mathrm{H}_{2}\right), 1.15\left(9 \mathrm{H}, \mathrm{s},{ }^{t} \mathrm{Bu}\right), 0.08\left(1 \mathrm{H}\right.$, ddd $\left.(\mathrm{J}=23.6,12.4,5.2), \mathrm{C}^{2} \mathrm{H}_{2}\right),-0.05(1 \mathrm{H}$, ddd $(\mathrm{J}=$ 
18.7, 10.1, 5.2), C1H); ${ }^{13} \mathrm{C}\left\{{ }^{1} \mathrm{H}\right\}$ NMR $\left(\mathrm{C}_{6} \mathrm{D}_{6}, 100 \mathrm{MHz}, 25^{\circ} \mathrm{C}, \delta\right): 154.2(\mathrm{C} 2), 122.7(\mathrm{C} 6), 109.7\left(\mathrm{Cp}^{*}\right.$ quat), 41.6 ('Bu quat), 39.8 (C4 or C5), 38.4 (C3), 35.9 (C4 or C5), $32.7\left({ }^{t} \mathrm{Bu} \mathrm{CH}_{3}\right), 20.4$ (1), 10.7 (Cp* $\left.\mathrm{CH}_{3}\right) . \mathrm{MS}\left(\mathrm{El}, 120^{\circ} \mathrm{C}\right): \mathrm{m} / \mathrm{z} 401\left[\mathrm{P}^{+}\right]$.

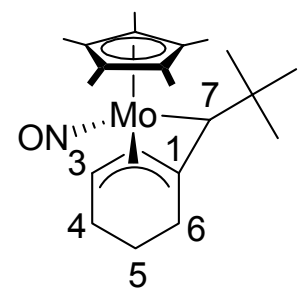

Complex 3C: A red solution of $\mathrm{Cp}^{*} \mathrm{Mo}(\mathrm{NO})\left(\mathrm{CH}_{2} \mathrm{CMe}_{3}\right)_{2}(0.047 \mathrm{~g}, 0.116$ $\mathrm{mmol})$ in cyclohexene $(6.64 \mathrm{~g})$ became brown after $110 \mathrm{~h}$. The solution was then evaporated to dryness in vacuo and extracted with pentane $(2 \times 2 \mathrm{~mL})$. The pentane extracts were then loaded onto the top of a column of neutral alumina $(2.5 \times 0.5 \mathrm{~cm})$ prepared with pentane. The column was washed with pentane $(15 \mathrm{~mL})$. A yellow band was then eluted with 9:1 pentane/diethyl ether $(15 \mathrm{~mL})$ and collected. The yellow eluate was evaporated to obtain $3 \mathrm{C}$ as a yellow solid $(0.018 \mathrm{~g}, 0.044 \mathrm{mmol}, 38 \%)$. X-ray quality crystals of $3 \mathrm{C}$ may be obtained by dissolving the compound in diethyl ether and slowly allowing the solvent to evaporate over a period of days at room temperature.

IR (Nujol): $v_{\mathrm{NO}}=1598 \mathrm{~cm}^{-1} ;{ }^{1} \mathrm{H}$ NMR $\left(\mathrm{C}_{6} \mathrm{D}_{6}, 500 \mathrm{MHz}, 25^{\circ} \mathrm{C}, \delta\right): 3.74(1 \mathrm{H}$, ddd $(J=6.7,6.7$, 1.0), $\mathrm{C} 3 \mathrm{H}), 2.52\left(1 \mathrm{H}, \mathrm{dd}(\mathrm{J}=16.5,4.9), \mathrm{C}^{2} \mathrm{H}_{2}\right), 2.30-2.40\left(3 \mathrm{H}, \mathrm{m}\right.$ (overlapping), $\mathrm{C} 4 \mathrm{H}_{2}$ (both), $\left.\mathrm{C}^{2} \mathrm{H}_{2}\right)$, $1.70(1 \mathrm{H}, \mathrm{s}, \mathrm{C} 7 \mathrm{H}), 1.65\left(15 \mathrm{H}, \mathrm{s}, \mathrm{Cp}^{*}\right), 1.54\left(2 \mathrm{H}, \mathrm{m}(\mathrm{J}=6.1), \mathrm{C}^{2} \mathrm{H}_{2}\right), 1.32\left(9 \mathrm{H}, \mathrm{s},{ }^{t} \mathrm{Bu}\right), 1.12(1 \mathrm{H}, \mathrm{d}(\mathrm{J}=$ 6.3), $\mathrm{C} 2 \mathrm{H}) ;{ }^{13} \mathrm{C}\left\{{ }^{1} \mathrm{H}\right\} \operatorname{NMR}\left(\mathrm{C}_{6} \mathrm{D}_{6}, 125 \mathrm{MHz}, 25^{\circ} \mathrm{C}, \delta\right): 115.8$ (C1), 105.2 (Cp* quat), 94.1 (C7), 85.9 (C2), 76.9 (C3), 34.9 ('Bu quat), 32.9 ( ${ }^{t} \mathrm{Bu} \mathrm{CH}$ ), 27.9 (C5), 26.8 (C4), 22.8 (C6), $10.8\left(\mathrm{Cp}^{*} \mathrm{CH}_{3}\right)$; MS (El, 120 $\left.{ }^{\circ} \mathrm{C}\right): m / z 413\left[\mathrm{P}^{+}\right]$

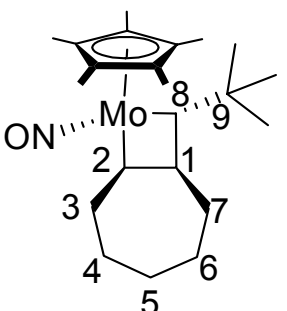

Complex 4A: A red solution of $\mathrm{Cp}^{*} \mathrm{Mo}(\mathrm{NO})\left(\mathrm{CH}_{2} \mathrm{CMe}_{3}\right)_{2}(0.036 \mathrm{~g}, 0.089$ $\mathrm{mmol})$ in cycloheptene $(0.485 \mathrm{~g})$ and cyclohexane $(4.1 \mathrm{~g})$ darkened to red brown after $6 \mathrm{~h}$. The solution was then evaporated to dryness in vacuo and the residue was taken into $\mathrm{C}_{6} \mathrm{D}_{6}$ for spectroscopic characterization as a mixture of $4 \mathrm{~A}$ and $4 \mathrm{~B}$.

IR (Nujol): $v_{\mathrm{NO}}=1588 \mathrm{~cm}^{-1} ;{ }^{1} \mathrm{H}$ NMR $\left(\mathrm{C}_{6} \mathrm{D}_{6}, 400 \mathrm{MHz}, 25^{\circ} \mathrm{C}\right.$, selected resonances only, $\left.\delta\right): 5.66$ (1H, dd $(J=12.7,9.9), \mathrm{C} 2 \mathrm{H}), 4.52(1 \mathrm{H}, \mathrm{d}(\mathrm{J}=4.2), \mathrm{C} 8 \mathrm{H}), 2.20\left(1 \mathrm{H}, \mathrm{br} \mathrm{d}(J=12.7), \mathrm{C}_{2} \mathrm{H}_{2}\right), 2.00(1 \mathrm{H}, \mathrm{m}$ (buried), $\left.\mathrm{C}^{\mathrm{H}} \mathrm{H}\right), 1.72\left(15 \mathrm{H}, \mathrm{s}, \mathrm{Cp}{ }^{*}\right), 1.60\left(1 \mathrm{H}, \mathrm{m}\right.$ (buried), $\left.\mathrm{C} \mathrm{H}_{2}\right), 1.22\left(9 \mathrm{H}, \mathrm{s},{ }^{t} \mathrm{Bu}\right), 0.41(1 \mathrm{H}, \mathrm{dd}(\mathrm{J}=$ 23.0, 11.0), $\left.\mathrm{C} \mathrm{H}_{2}\right),-0.46(1 \mathrm{H}, \mathrm{dd}(J=9.9,4.2), \mathrm{C} 1 \mathrm{H}) ;{ }^{13} \mathrm{C}\left\{{ }^{1} \mathrm{H}\right\}$ NMR $\left(\mathrm{C}_{6} \mathrm{D}_{6}, 100 \mathrm{MHz}, 25{ }^{\circ} \mathrm{C}\right.$, selected resonances): 155.5 (C8), 110.2 (C2), 109.6 (Cp* quat), 36.8 (C3), 32.5 ('Bu $\left.\mathrm{CH}_{3}\right), 31.9$ (7), 10.9 (Cp* $\left.\mathrm{CH}_{3}\right)$. 


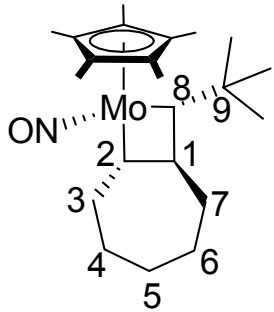

Complex 4B: A red solution of $\mathrm{Cp}{ }^{*} \mathrm{Mo}(\mathrm{NO})\left(\mathrm{CH}_{2} \mathrm{CMe}_{3}\right)_{2}(0.041 \mathrm{~g}, 0.102$ $\mathrm{mmol})$ in cycloheptene $(0.521 \mathrm{~g})$ and cyclohexane $(8 \mathrm{~g})$ turned brown after $20 \mathrm{~h}$. The final solution was then evaporated to dryness in vacuo. The resulting residue was dissolved in pentane $(2 \mathrm{~mL})$ and the solvent was allowed to slowly evaporate over 2 days to give dark red crystals which were washed with small aliquots of cold pentane to obtain 4B $(0.011 \mathrm{~g}, 0.026 \mathrm{mmol}, 25 \%)$.

IR (Nujol): $v_{\mathrm{NO}}=1586 \mathrm{~cm}^{-1} ;{ }^{1} \mathrm{H}$ NMR $\left(\mathrm{C}_{6} \mathrm{D}_{6}, 400 \mathrm{MHz}, 25{ }^{\circ} \mathrm{C}, \delta\right): 4.34(1 \mathrm{H}$, ddd $(J=9.2,5.3$, 5.3), $\mathrm{C} 2 \mathrm{H}), 3.71(1 \mathrm{H}, \mathrm{d}(\mathrm{J}=6.1), \mathrm{C} 8 \mathrm{H}), 2.70\left(2 \mathrm{H}, \mathrm{m}, \mathrm{C} 3 \mathrm{H}_{2}\right), 2.35(1 \mathrm{H}$, dddd $(J=13.3,6.3,6.3,2.4)$, $\left.\mathrm{C} \mathrm{H}_{2}\right), 1.70\left(15 \mathrm{H}, \mathrm{s}, \mathrm{Cp}^{*} \mathrm{CH}_{3}\right), 1.70\left(1 \mathrm{H}, \mathrm{m}\right.$ (under $\left.\left.\mathrm{Cp}^{*}\right), \mathrm{C} 4 \mathrm{H}_{2}\right), 1.60\left(2 \mathrm{H}, \mathrm{m}\right.$ (overlapping), $\mathrm{C}_{4} \mathrm{H}_{2}$, $\left.\mathrm{C}^{2} \mathrm{H}_{2}\right), 1.58\left(1 \mathrm{H}, \mathrm{m}\right.$ (overlapping), $\left.\mathrm{C}^{2} \mathrm{H}_{2}\right), 1.54\left(1 \mathrm{H}, \mathrm{m}\right.$ (overlapping), $\left.\mathrm{C}^{2} \mathrm{H}_{2}\right), 1.43(1 \mathrm{H}, \mathrm{m}$ (overlapping), $\left.\mathrm{C}^{\mathrm{CH}} \mathrm{H}_{2}\right), 1.27\left(9 \mathrm{H}, \mathrm{s},{ }^{t} \mathrm{Bu} \mathrm{CH}_{3}\right), 0.35\left(1 \mathrm{H}\right.$, ddd $\left.(\mathrm{J}=20.0,13.3,6.3), \mathrm{C} \mathrm{H}_{2}\right),-0.28(1 \mathrm{H}$, dddd $(J=20.0,9.2$, 6.1, 2.4), $\mathrm{C} 1 \mathrm{H}) ;{ }^{13} \mathrm{C}\left\{{ }^{1} \mathrm{H}\right\}$ NMR $\left(\mathrm{C}_{6} \mathrm{D}_{6}, 100 \mathrm{MHz}, 25{ }^{\circ} \mathrm{C}, \delta\right): 138.2$ (C8), 130.9 (C2), 109.3 (Cp* quat), 41.6 (C3), 32.9 ('Bu CH 3 ), 32.7 (C4), 31.5 (C6), 30.0 (C7), 25.2 (C5), 15.2 (C1), $10.7\left(\mathrm{Cp}^{*} \mathrm{CH}_{3}\right) . \mathrm{MS}$ (El, 120 $\left.{ }^{\circ} \mathrm{C}\right): m / z 427\left[\mathrm{P}-2^{+}\right]$.

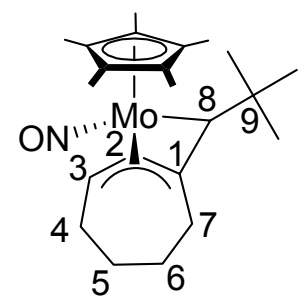

Complex 4C: A red solution of $\mathrm{Cp}^{*} \mathrm{Mo}(\mathrm{NO})\left(\mathrm{CH}_{2} \mathrm{CMe}_{3}\right)_{2}(0.062 \mathrm{~g}, 0.154$ $\mathrm{mmol})$ in cycloheptene $(0.835 \mathrm{~g})$ and cyclohexane $(7.2 \mathrm{~g})$ was heated at $50{ }^{\circ} \mathrm{C}$ for 20 h. The solution was then evaporated to dryness in vacuo and extracted with pentane $(2 \times 1 \mathrm{~mL})$. The pentane extracts were then loaded onto the top of a column of neutral alumina $(3 \times 1 \mathrm{~cm})$ prepared with pentane. The column was first washed with pentane $(3 \mathrm{~mL})$. A yellow band was then eluted with 3:1 pentane/diethyl ether $(6 \mathrm{~mL})$ and collected. The yellow eluate was evaporated in vacuo and washed with cold pentane to obtain $\mathbf{4 C}$ as a yellow solid $(0.023 \mathrm{~g}, 0.054$ mmol, $35 \%)$.

IR (Nujol): $v_{\text {NO }}=1589 \mathrm{~cm}^{-1} ;{ }^{1} \mathrm{H}$ NMR $\left(\mathrm{C}_{6} \mathrm{D}_{6}, 400 \mathrm{MHz}, 25^{\circ} \mathrm{C}, \delta\right): 3.21(1 \mathrm{H}$, ddd $(J=9.0,4.5$, 3.0), C3H), $2.90\left(1 \mathrm{H}, \mathrm{d}(\mathrm{J}=17.1), \mathrm{C} 7 \mathrm{H}_{2}\right), 2.72\left(1 \mathrm{H}, \mathrm{m}, \mathrm{C} 4 \mathrm{H}_{2}\right), 2.61\left(1 \mathrm{H}, \mathrm{m}, \mathrm{C} 7 \mathrm{H}_{2}\right), 2.42(1 \mathrm{H}, \mathrm{d}(\mathrm{J}=$ 15.2), $\mathrm{C} 4 \mathrm{H}_{2}$ ), $1.68\left(1 \mathrm{H}, \mathrm{m}\right.$ (overlapping), $\mathrm{C}_{5} \mathrm{H}_{2}$ or $\left.\mathrm{C} 6 \mathrm{H}_{2}\right), 1.67$ (1H, m (overlapping), $\mathrm{C} 5 \mathrm{H}_{2}$ or $\mathrm{C}_{6} \mathrm{H}_{2}$ ), $1.66\left(1 \mathrm{H}, \mathrm{m}\right.$ (overlapping), $\mathrm{C}_{5} \mathrm{H}_{2}$ or $\left.\mathrm{C}^{2} \mathrm{H}_{2}\right), 1.64\left(15 \mathrm{H}, \mathrm{s}, \mathrm{Cp} \mathrm{p}^{*}\right), 1.63\left(1 \mathrm{H}, \mathrm{m}\right.$ (overlapping), $\mathrm{C}^{2} \mathrm{H}_{2}$ or $\left.\mathrm{C}^{2} \mathrm{H}_{2}\right), 1.40(1 \mathrm{H}, \mathrm{s}, \mathrm{C} 8 \mathrm{H}), 1.33\left(9 \mathrm{H}, \mathrm{s},{ }^{t} \mathrm{Bu}\right), 0.21(1 \mathrm{H}, \mathrm{d}(\mathrm{J}=9.0), \mathrm{C} 2 \mathrm{H}) ;{ }^{13} \mathrm{C}\left\{{ }^{1} \mathrm{H}\right\} \mathrm{NMR}\left(\mathrm{C}_{6} \mathrm{D}_{6}, 100 \mathrm{MHz}\right.$, 
$25{ }^{\circ} \mathrm{C}, \delta$ ): 123.2 (C1), 105.5 (Cp* quat), 93.6 (C8), 82.2 (C3), 81.1 (C2), 35.5 ('Bu quat), 33.3 (C7), 33.0 ( ${ }^{t} \mathrm{Bu} \mathrm{CH} \mathrm{CH}_{3}$ ), 33.0 (C4), 26.5 (C5 or C6), 26.4 (C5 or C6), $10.9\left(\mathrm{Cp}^{*} \mathrm{CH}_{3}\right) . \mathrm{MS}$ (El, $\left.100{ }^{\circ} \mathrm{C}\right): \mathrm{m} / \mathrm{z} 427$

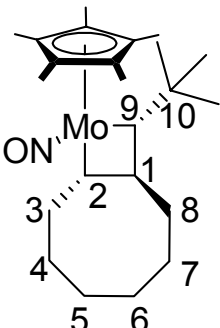
$\left[\mathrm{P}^{+}\right]$

Complex 5B: A red solution of $\mathrm{Cp}{ }^{*} \mathrm{Mo}(\mathrm{NO})\left(\mathrm{CH}_{2} \mathrm{CMe}_{3}\right)_{2}(0.063 \mathrm{~g}, 0.156 \mathrm{mmol})$ in cyclooctene $(0.890 \mathrm{~g})$ was diluted with cyclohexane $(10.2 \mathrm{~g})$. The solution darkened to brown after $18 \mathrm{~h}$. The solution was then evaporated to dryness in vacuo and extracted with pentane $(2 \times 1 \mathrm{~mL})$. The pentane extracts were then loaded onto the top of a column of neutral alumina $(3 \times 1 \mathrm{~cm})$ prepared with pentane. The column was washed with pentane $(3 \mathrm{~mL})$ and then an orange band was eluted using 5:1 pentane/diethyl ether $(6 \mathrm{~mL})$ and collected. The orange eluate was evaporated to dryness in vacuo, dissolved in minimal pentane, and stored at $-30{ }^{\circ} \mathrm{C}$ overnight to obtain 5B as red crystals $(0.035 \mathrm{~g}, 0.079 \mathrm{mmol}, 51 \%)$.

Anal. Calcd for $\mathrm{C}_{23} \mathrm{H}_{39} \mathrm{MoNO}$ : C, 62.57; H, 8.90; N, 3.17. Found: C, 62.81; $\mathrm{H}, 8.60 ; \mathrm{N}, 3.46$; IR (Nujol): $v_{\mathrm{NO}}=1586 \mathrm{~cm}^{-1} ;{ }^{1} \mathrm{H}$ NMR $\left(\mathrm{C}_{6} \mathrm{D}_{6}, 400 \mathrm{MHz}, 25^{\circ} \mathrm{C}, \delta\right): 4.31(1 \mathrm{H}, \mathrm{d}(\mathrm{J}=4.4), \mathrm{C} 9 \mathrm{H}), 4.10(1 \mathrm{H}, \mathrm{ddd}$ $(J=11.7,4.0,3.4)), \mathrm{C} 2 \mathrm{H}), 2.79\left(1 \mathrm{H}, \mathrm{ddd}(\mathrm{J}=14.3,5.8,3.0), \mathrm{C} 3 \mathrm{H}_{2}\right), 2.45\left(1 \mathrm{H}, \mathrm{m}, \mathrm{C} 3 \mathrm{H}_{2}\right), 2.19(1 \mathrm{H}, \mathrm{m}$, $\left.\mathrm{C} \mathrm{H}_{2}\right), 1.82\left(1 \mathrm{H}, \mathrm{m}\right.$ (overlapping), $\left.\mathrm{C} 4 \mathrm{H}_{2}\right), 1.73\left(1 \mathrm{H}, \mathrm{m}\right.$ (overlapping), $\left.\mathrm{C}^{2} \mathrm{H}_{2}\right), 1.69\left(15 \mathrm{H}, \mathrm{s}, \mathrm{Cp} \mathrm{p}^{*}\right), 1.64$ (1 $\mathrm{H}, \mathrm{m}$ (overlapping), $\mathrm{C} 7 \mathrm{H}_{2}$ ), $1.51\left(1 \mathrm{H}, \mathrm{m}\right.$ (overlapping), $\left.\mathrm{C}_{4} \mathrm{H}_{2}\right), 1.50\left(1 \mathrm{H}, \mathrm{m}\right.$ (overlapping), $\mathrm{C} 5 \mathrm{H}_{2}$ ), 1.48 (1H, m (overlapping), $\left.\mathrm{C} \mathrm{H}_{2}\right), 1.46\left(1 \mathrm{H}, \mathrm{m}\right.$ (overlapping), $\left.\mathrm{C}^{2} \mathrm{H}_{2}\right), 1.36\left(1 \mathrm{H}, \mathrm{m}\right.$ (overlapping), $\left.\mathrm{C} 6 \mathrm{H}_{2}\right), 1.28$ $\left(9 \mathrm{H}, \mathrm{s},{ }^{t} \mathrm{Bu}\right), 0.20\left(1 \mathrm{H}, \mathrm{m}, \mathrm{C} 8 \mathrm{H}_{2}\right),-0.18(1 \mathrm{H}, \mathrm{ddd}(\mathrm{J}=11.7,7.5,4.4), \mathrm{C} 1 \mathrm{H}) ;{ }^{13} \mathrm{C}\left\{{ }^{1} \mathrm{H}\right\} \mathrm{NMR}\left(\mathrm{C}_{6} \mathrm{D}_{6}, 100\right.$ $\left.\mathrm{MHz}, 25^{\circ} \mathrm{C}, \delta\right): 152.3$ (C9), 121.5 (C2), 109.3 (Cp* quat), 44.4 (C3), 44.1 ('Bu quat), 36.1 (C8), 32.9 ( ${ }^{t} \mathrm{Bu} \mathrm{CH}_{3}$ ), 30.5 (C4), 28.8 (C7), 28.2 (C5 or C6), 28.1 (C5 or C6), $14.9(\mathrm{C} 1), 10.7\left(\mathrm{Cp}^{*} \mathrm{CH}_{3}\right)$; MS (El,

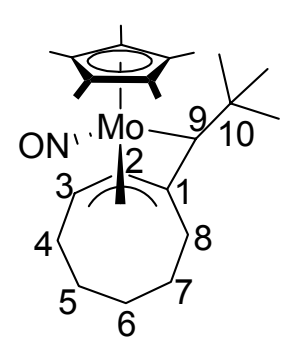
$\left.100{ }^{\circ} \mathrm{C}\right): m / z 441\left[\mathrm{P}-2^{+}\right]$.

Complex 5C: An orange solution of 5B $(0.030 \mathrm{~g}, 0.068 \mathrm{mmol})$ in cyclohexane $(8.0 \mathrm{~g})$ was heated at $50{ }^{\circ} \mathrm{C}$ for $48 \mathrm{~h}$. The solution was then evaporated to dryness in vacuo and extracted with pentane $(2 \times 1 \mathrm{~mL})$. The pentane extracts were then loaded onto the top of a column of neutral alumina $(2 \times 1 \mathrm{~cm})$ prepared with pentane. The column was washed with pentane $(3 \mathrm{~mL})$ and then a yellow band was eluted using 5:1 pentane/diethyl ether $(6 \mathrm{~mL})$ and collected. The yellow eluate was evaporated in vacuo to obtain $\mathbf{5 C}$ as a yellow solid (0.015 g, $0.034 \mathrm{mmol}, 50 \%)$. 
Anal. Calcd for $\mathrm{C}_{23} \mathrm{H}_{37} \mathrm{MoNO}$ : C, 62.86; H, 8.49; N, 3.19; Found: C, 62.45; H, 8.59; N, 3.38;

IR (Nujol): $v_{\mathrm{NO}}=1595 \mathrm{~cm}^{-1} ;{ }^{1} \mathrm{H}$ NMR $\left(\mathrm{C}_{6} \mathrm{D}_{6}, 400 \mathrm{MHz}, 25^{\circ} \mathrm{C}, \delta\right): 3.06(1 \mathrm{H}$, ddd $(J=16.3,10.3,8.0)$, $\mathrm{C} 3 \mathrm{H}), 2.83\left(1 \mathrm{H}\right.$, ddd $\left.(\mathrm{J}=17.1,5.9,3.9), \mathrm{C} \mathrm{H}_{2}\right), 2.33\left(2 \mathrm{H}, \mathrm{m}\right.$ (overlapping), $\left.\mathrm{C}_{4} \mathrm{H}_{2}, \mathrm{C} \mathrm{H}_{2}\right), 2.21(1 \mathrm{H}, \mathrm{m}$ (overlapping), $\left.\mathrm{C}^{2} \mathrm{H}_{2}\right), 2.14\left(1 \mathrm{H}, \mathrm{m}\right.$ (overlapping), $\left.\mathrm{C}^{2} \mathrm{H}_{2}\right), 1.89\left(1 \mathrm{H}, \mathrm{m}, \mathrm{C} 7 \mathrm{H}_{2}\right), 1.81(1 \mathrm{H}, \mathrm{m}$ (overlapping), $\left.\mathrm{C}^{2} \mathrm{H}_{2}\right), 1.75\left(1 \mathrm{H}, \mathrm{m}\right.$ (overlapping), $\left.\mathrm{C}^{2} \mathrm{H}_{2}\right), 1.67\left(15 \mathrm{H}, \mathrm{s}, \mathrm{Cp}^{*} \mathrm{CH}_{3}\right), 1.66(1 \mathrm{H}, \mathrm{s}, \mathrm{C} 9 \mathrm{H}), 1.57(1 \mathrm{H}, \mathrm{m}$, $\left.\mathrm{C}^{\mathrm{H}} \mathrm{H}_{2}\right), 1.40\left(1 \mathrm{H}, \mathrm{m}, \mathrm{C}_{6} \mathrm{H}_{2}\right), 1.30\left(9 \mathrm{H}, \mathrm{s},{ }^{t} \mathrm{Bu} \mathrm{CH}_{3}\right), 0.72(1 \mathrm{H}, \mathrm{d}(\mathrm{J}=8.0), \mathrm{C} 2 \mathrm{H}) \cdot{ }^{13} \mathrm{C}\left\{{ }^{1} \mathrm{H}\right\} \operatorname{NMR}\left(\mathrm{C}_{6} \mathrm{D}_{6}, 100\right.$ $\mathrm{MHz}, 25^{\circ} \mathrm{C}, \delta$ ): 121.3 (C1), 105.6 (Cp* quat), 96.7 (C9), 85.2 (C2), 78.0 (C3), 34.9 ('Bu quat), 33.3 ( ${ }^{t} \mathrm{Bu}$ $\mathrm{CH}_{3}$ ), 31.8 (C5), 31.3 (C4), 30.1 (C7), 29.0 (C8), 28.0 (C6), $11.3\left(\mathrm{Cp}^{*} \mathrm{CH}_{3}\right) ; \mathrm{MS}$ (El, $\left.100{ }^{\circ} \mathrm{C}\right): \mathrm{m} / \mathrm{z} 441$ $\left[\mathrm{P}^{+}\right]$.

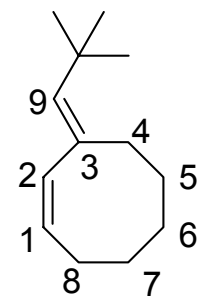

3-Neopentylidene-cyclooctene (6): A yellow solution of 5C (0.021 g, $0.048 \mathrm{mmol})$ in cyclopentene $(2.0 \mathrm{~g})$ was heated at $70{ }^{\circ} \mathrm{C}$ for $3 \mathrm{~d}$. The reaction mixture was then evaporated to dryness in vacuo and extracted with pentane $(2 \times 1 \mathrm{~mL})$. The pentane extracts were then loaded onto the top of a column of neutral alumina $(2 \times 1 \mathrm{~cm})$ prepared with pentane. The column was eluted with pentane and all the pentane eluates were collected and evaporated in vacuo to obtain 6 as a colorless oil $(0.04 \mathrm{~g}, 0.022 \mathrm{mmol}, 47 \%)$.

${ }^{1} \mathrm{H}$ NMR $\left(\mathrm{C}_{6} \mathrm{D}_{6}, 400 \mathrm{MHz}, 25{ }^{\circ} \mathrm{C}, \delta\right): 6.21(1 \mathrm{H}, \mathrm{d}(\mathrm{J}=12.0), \mathrm{C} 2 \mathrm{H}), 5.57(1 \mathrm{H}, \mathrm{s}, \mathrm{C} 9 \mathrm{H}), 5.37(1 \mathrm{H}$, ddd $(J=12.0,8.6,3.4), \mathrm{C} 1 \mathrm{H}), 2.64\left(2 \mathrm{H}, \mathrm{t}(J=6.5), \mathrm{C} \mathrm{H}_{2}(\mathrm{both})\right), 2.33\left(2 \mathrm{H}, \mathrm{dd}(J=14.9,8.6), \mathrm{C}^{2} \mathrm{H}_{2}\right.$ (both)), $1.62\left(4 \mathrm{H}, \mathrm{m}, \mathrm{C} 5 \mathrm{H}_{2}\right.$ (both), $\mathrm{C} \mathrm{H}_{2}$ (both)), $1.48\left(2 \mathrm{H}, \mathrm{m}, \mathrm{C} 7 \mathrm{H}_{2}\right.$ (both)), $1.09\left(9 \mathrm{H}, \mathrm{s},{ }^{t} \mathrm{Bu}\right) ;{ }^{13} \mathrm{C}\left\{{ }^{1} \mathrm{H}\right\}$ $\operatorname{NMR}\left(\mathrm{C}_{6} \mathrm{D}_{6}, 100 \mathrm{MHz}, 25^{\circ} \mathrm{C}, \delta\right): 144.5$ (C9), 140.3 (C2), 138.6 (C3), 124.8 (C1), $32.0\left({ }^{t} \mathrm{Bu} \mathrm{CH} \mathrm{CH}_{3}\right), 28.6$ (C7), 28.2 ('Bu quat), 28.1 (C4), 26.5 (C8), 23.2 (C5 or C6), 23.1 (C5 or C 6); MS (El, $100{ }^{\circ} \mathrm{C}$ ): $\mathrm{m} / \mathrm{z} 178$ $\left[\mathrm{P}^{+}\right]$.

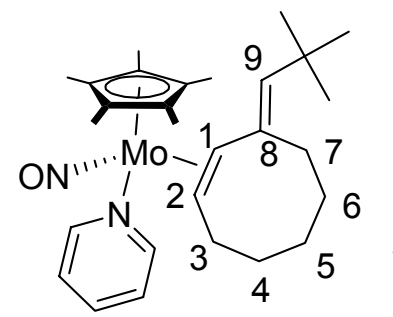

Complex 7: A yellow solution of $5 \mathrm{C}(0.027 \mathrm{~g}, 0.061 \mathrm{mmol})$ in pyridine $(3.2 \mathrm{~g})$ was heated at $100^{\circ} \mathrm{C}$ for $19 \mathrm{~h}$. The reaction mixture was then evaporated to dryness in vacuo and extracted with pentane $(2 \times 1 \mathrm{~mL})$. The pentane extracts were then loaded onto the top of a column of neutral alumina $(2 \times 1 \mathrm{~cm})$ prepared with pentane. The column was washed with 3:1 pentane/diethyl ether $(3 \mathrm{~mL})$ to elute any remaining starting material as a yellow band. Next, a red orange band was eluted using 1:3 pentane/diethyl ether $(6 \mathrm{~mL})$ and collected. This eluate was evaporated in vacuo to give a red residue 
which was dissolved in minimal pentane and stored at $-30{ }^{\circ} \mathrm{C}$ overnight to obtain 7 as an orange precipitate $(0.023 \mathrm{~g}, 0.044 \mathrm{mmol}, 72 \%)$.

Anal. Calcd for $\mathrm{C}_{28} \mathrm{H}_{42} \mathrm{MoN}_{2} \mathrm{O}$ : C, 64.85; $\mathrm{H}, 8.16$; N, 5.40; Found: C, 64.84; H, 8.49; N, 5.80; IR (Nujol): $v_{\mathrm{NO}}=1552 \mathrm{~cm}^{-1} ;{ }^{1} \mathrm{H}$ NMR $\left(\mathrm{C}_{6} \mathrm{D}_{6}, 400 \mathrm{MHz}, 25^{\circ} \mathrm{C}, \delta\right): 8.07(2 \mathrm{H}, \mathrm{br} \mathrm{s}$, ortho py), $6.64(1 \mathrm{H}, \mathrm{t}(\mathrm{J}=$ 7.5), para py), $6.39\left(2 \mathrm{H}, \mathrm{t}(\mathrm{J}=6.4)\right.$, meta py), $4.80(1 \mathrm{H}, \mathrm{s}, \mathrm{C} 9 \mathrm{H}), 3.13\left(1 \mathrm{H}, \mathrm{m}, \mathrm{C} 3 \mathrm{H}_{2}\right), 2.81(1 \mathrm{H}, \mathrm{m}$, C7 $\left.\mathrm{H}_{2}\right), 2.57\left(1 \mathrm{H}, \mathrm{m}, \mathrm{C} 4 \mathrm{H}_{2}\right), 2.45\left(1 \mathrm{H}, \mathrm{dd}(\mathrm{J}=13.7,8.6), \mathrm{C}^{2} \mathrm{H}_{2}\right), 2.27\left(1 \mathrm{H}, \mathrm{m}, \mathrm{C} 3 \mathrm{H}_{2}\right), 1.93(2 \mathrm{H}, \mathrm{m}$ (buried), $\mathrm{C} \mathrm{H}_{2}$ (both)), $1.90(1 \mathrm{H}$, buried, $\mathrm{C} 1 \mathrm{H}), 1.87(1 \mathrm{H}$, buried, $\mathrm{C} 2 \mathrm{H}), 1.75\left(1 \mathrm{H}, \mathrm{m}, \mathrm{C} 4 \mathrm{H}_{2}\right), 1.64(1 \mathrm{H}$, m, C5H $\left.\mathrm{H}_{2}\right), 1.53(15 \mathrm{H}, \mathrm{s}, \mathrm{Cp}), 1.37\left(1 \mathrm{H}, \mathrm{m}, \mathrm{C} 5 \mathrm{H}_{2}\right), 0.95\left(9 \mathrm{H}, \mathrm{s},{ }^{t} \mathrm{Bu}\right) ;{ }^{13} \mathrm{C}\left\{{ }^{1} \mathrm{H}\right\} \mathrm{NMR}\left(\mathrm{C}_{6} \mathrm{D}_{6}, 100 \mathrm{MHz}, 25\right.$ ${ }^{\circ} \mathrm{C}, \delta$ ): 154.6 (ortho py), 145.1 (C8 (observed by HMBC)), 135.0 (para py), 129.3 (C9), 124.6 (meta py), 105.4 (Cp* quat), 77.1 (C1), 61.9 (C2), 34.7 (C6), 33.1 ('Bu $\mathrm{CH}_{3}$ ), 32.8 (C5), 32.2 (C3), 28.0 ('Bu quat), 26.2 (C7), 23.1 (C4), $9.7\left(\mathrm{Cp}^{*} \mathrm{CH}_{3}\right)$; $\mathrm{MS}\left(\mathrm{El}, 120^{\circ} \mathrm{C}\right): \mathrm{m} / \mathrm{z} 520\left[\mathrm{P}^{+}\right]$.

X-ray Crystallography. Data collection for each compound was carried out at $-100 \pm 1{ }^{\circ} \mathrm{C}$ on a Rigaku AFC7/ADSC CCD diffractometer, using graphite-monochromated Mo Ka radiation.

Data for $2 \mathrm{~A}$ were collected to a maximum $2 \theta$ value of $55.2^{\circ}$ in $0.5^{\circ}$ oscillations. The structure was solved by direct methods ${ }^{2}$ and expanded using Fourier techniques. All non-hydrogen atoms were refined anisotropically and all hydrogen atoms were included in fixed positions. The final cycle of fullmatrix least-squares analysis was based on 3600 observed reflections and 216 variable parameters.

Data for $3 \mathrm{C}$ were collected to a maximum $2 \theta$ value of $55.0^{\circ}$ in $0.5^{\circ}$ oscillations. The structure was solved by direct methods ${ }^{2}$ and expanded using Fourier techniques. The coupled organic ligand and the nitrosyl ligand are disordered in two orientations. Restraints were used to maintain similar geometries for the related segments, namely the tert-butyl groups, the cyclohexane rings, and the nitrosyl ligands. Overlapping atoms ( $\mathrm{N} 1$ and $\mathrm{C} 23, \mathrm{~N} 2$ and $\mathrm{C} 2, \mathrm{C} 8$ and $\mathrm{C} 29$, and $\mathrm{C} 11$ and $\mathrm{C} 32$ ) were refined isotropically; all other non-hydrogen atoms were refined anisotropically. Hydrogen atoms $\mathrm{H} 2$, $\mathrm{H} 3, \mathrm{H} 23$, and $\mathrm{H} 24$ were refined isotropically, and all other hydrogen atoms were included in fixed positions. The final cycle of full-matrix least-squares analysis was based on 4157 observed reflections and 322 variable parameters.

Data for $5 \mathrm{~B}$ were collected to a maximum $2 \theta$ value of $49.8^{\circ}$ in $0.5^{\circ}$ oscillations. The structure was solved by direct methods ${ }^{2}$ and expanded using Fourier techniques. All non-hydrogen atoms were 
refined anisotropically; hydrogen atoms $\mathrm{H} 1, \mathrm{H} 2$, and $\mathrm{H} 9$ were refined isotropically, and all other hydrogen atoms were included in fixed positions. The final cycle of full-matrix least-squares analysis was based on 3966 observed reflections and 255 variable parameters.

For each structure neutral-atom scattering factors were taken from Cromer and Waber. ${ }^{3}$ Anomalous dispersion effects were included in $F_{\text {calc }}{ }^{4}$ the values for $\Delta f$ and $\Delta f^{\prime}$ were those of Creagh and McAuley. ${ }^{5}$ The values for mass attenuation coefficients are those of Creagh and Hubbell. ${ }^{6}$ All calculations were performed using the CrystalClear software package of Rigaku/MSC, ${ }^{7}$ or Shelxl-97. ${ }^{8}$ X-ray crystallographic data for the three structures are presented in Table 1, and in the cif files. 
Table 1. X-ray Crystallographic Data for Complexes 2A, 3C and 5B

2A $\quad 3$ C

Crystal Data

Empirical formula

Crystal Habit, color

$\mathrm{C}_{20} \mathrm{H}_{33} \mathrm{NOMo}$

$\mathrm{C}_{21} \mathrm{H}_{33} \mathrm{NOMo}$

$\mathrm{C}_{23} \mathrm{H}_{39} \mathrm{NOMo}$

Prism, orange

$0.5 \times 0.3 \times 0.1$

Irregular, yellow

Irregular, red-orange

Crystal size (mm)

Crystal system

Triclinic

$0.50 \times 0.50 \times 0.25$

$0.25 \times 0.20 \times 0.20$

Space group

Volume $\left(\AA^{3}\right)$

P-1

Monoclinic

Monoclinic

$\mathrm{P} 2{ }_{1} / \mathrm{c}$

$\mathrm{C} 2 / \mathrm{c}$

945.7(2)

1997.1(4)

4561.5(8)

$8.3346(8)$

8.934(1)

19.614(2)

8.4671(8)

$13.776(2)$

17.114(2)

14.507(1)

$84.830(5)$

$85.483(5)$

68.227(4)

2

$16.5414(8)$

15.519(2)

90

90

$\beta\left(^{\circ}\right)$

$\gamma\left({ }^{\circ}\right)$

Z

Density (calculated) $\left(\mathrm{Mg} / \mathrm{m}^{3}\right)$

1.403

6.98

420

101.191(7)

90

118.880(4)

90

4

8

Absorption coefficient $\left(\mathrm{cm}^{-1}\right)$

$\mathrm{F}_{000}$

Data Collection and Refinement

Measured Reflections: Total

6332

7285

7526

Measured Reflections: Unique

3600

4157

3966

Final R Indices ${ }^{\mathrm{a}}$

$\mathrm{R} 1=0.0204, \mathrm{wR} 2=$

1.286

6.64

5.86

1872

864

$\mathrm{R} 1=0.0253, \mathrm{wR} 2=$

$$
0.0535
$$

0.0713 0.0591

Goodness-of-fit on $\mathrm{F}^{2 \mathrm{~b}}$

1.068

1.069

1.021

Largest diff. peak and hole $\left(\mathrm{e}^{-} \AA^{-3}\right) \quad 0.354$ and -0.456

0.440 and -0.445

0.308 and -0.250

${ }^{\mathrm{a}} \mathrm{R} 1$ on $\mathrm{F}=\Sigma\left|\left(\left|\mathrm{F}_{\mathrm{o}}\right|-\left|\mathrm{F}_{\mathrm{c}}\right|\right)\right| / \Sigma\left|\mathrm{F}_{\mathrm{o}}\right|,(\mathrm{I}>2 \sigma(\mathrm{I})) ; \mathrm{wR} 2=\left[\left(\Sigma\left(\mathrm{F}_{\mathrm{o}}{ }^{2}-\mathrm{F}_{\mathrm{c}}{ }^{2}\right)^{2}\right) / \Sigma \mathrm{w}\left(\mathrm{F}_{\mathrm{o}}{ }^{2}\right)^{2}\right]^{1 / 2}$ (all data); w $=[$ $\left.\sigma^{2} \mathrm{~F}_{\mathrm{o}}{ }^{2}\right]^{-1} ;{ }^{\mathrm{b}} \mathrm{GOF}=\left[\Sigma\left(\mathrm{w}\left(\left|\mathrm{F}_{\mathrm{o}}\right|-\left|\mathrm{F}_{\mathrm{c}}\right|\right)^{2}\right) / \text { degrees of freedom }\right]^{1 / 2}$. 
References and Notes.

(1) Wada, K.; Pamplin, C. B.; Legzdins, P.; Patrick, B. O.; Tsyba,I.; Bau, R. J. Am. Chem. Soc. 2003, $125,7035-7048$.

(2) SIR92: Altomare, A.; Cascarano, M.; Giacovazzo, C.; Guagliardi, A. J. Appl. Cryst. 1993, 26, 343.

(3) Cromer, D. T.; Waber, J. T. International Tables for X-ray Crystallography; Kynoch Press:

Birmingham, 1974; Vol. IV.

(4) Ibers, J. A.; Hamilton, W. C. Acta Crystallogr. 1964, 17, 781-782.

(5) Creagh, D. C.; McAuley, W. J. International Tables of X-ray Crystallography; Kluwer Academic Publishers: Boston, 1992; Vol. C.

(6) Creagh, D. C.; Hubbell, J. H. International Tables for X-ray Crystallography; Kluwer Academic Publishers: Boston, 1992; Vol. C.

(7) CrystalClear: Version1.3.5b20; Molecular Structure Corporation, 2002.

(8) SHELXL97: Sheldrick, G. M. University of Göttingen, Germany, 1997. 